\title{
Higher Reliance on Glycolysis Limits Glycolytic Responsiveness in Degenerating Glaucomatous Optic Nerve
}

\author{
Assraa Hassan Jassim ${ }^{1}$ - Lucy Coughlin ${ }^{1}$. Mohammad Harun-Or-Rashid ${ }^{1}$ Patrick T. Kang ${ }^{2} \cdot$ Yeong-Renn Chen ${ }^{2}$. \\ Denise M. Inman ${ }^{1}$
}

Received: 11 December 2018 / Accepted: 20 March 2019 / Published online: 13 April 2019

(C) The Author(s) 2019

\begin{abstract}
Metabolic dysfunction accompanies neurodegenerative disease and aging. An important step for therapeutic development is a more sophisticated understanding of the source of metabolic dysfunction, as well as to distinguish disease-associated changes from aging effects. We examined mitochondrial function in ex vivo aging and glaucomatous optic nerve using a novel approach, the Seahorse Analyzer. Optic nerves (ON) from the DBA/2J mouse model of glaucoma and the DBA/2-Gpnmb $b^{+}$control strain were isolated, and oxygen consumption rate (OCR) and extracellular acidification rate (ECAR), the discharge of protons from lactate release or byproducts of substrate oxidation, were measured. The glial-specific aconitase inhibitor fluorocitrate was used to limit the contribution of glial mitochondria to OCR and ECAR. We observed significant decreases in maximal respiration, ATP production, and spare capacity with aging. In the presence of fluorocitrate, OCR was higher, with more ATP produced, in glaucoma compared to aged ON. However, glaucoma ON showed lower maximal respiration. In the presence of fluorocitrate and challenged with ATPase inhibition, glaucoma ON was incapable of further upregulation of glycolysis to compensate for the loss of oxidative phosphorylation. Inclusion of 2-deoxyglucose as a substrate during ATPase inhibition indicated a significantly higher proportion of ECAR was derived from TCA cycle substrate oxidation than glycolysis in glaucoma ON. These data indicate that glaucoma axons have limited ability to respond to increased energy demand given their lower maximal respiration and inability to upregulate glycolysis when challenged. The higher ATP output from axonal mitochondria in glaucoma optic nerve compensates for this lack of resiliency but is ultimately inadequate for continued function.
\end{abstract}

Keywords Seahorse analyzer $\cdot$ Fluorocitrate $\cdot \mathrm{DBA} / 2 \mathrm{~J} \cdot$ Glaucoma $\cdot$ Mitochondria $\cdot$ Optic nerve

\section{Abbreviations}

AA antimycin A

AD Alzheimer's disease

AMPK adenosine monophosphate activated protein kinase

Assraa Hassan Jassim and Lucy Coughlin are co-first authors.

Electronic supplementary material The online version of this article (https://doi.org/10.1007/s12035-019-1576-4) contains supplementary material, which is available to authorized users.

Denise M. Inman

dinman@neomed.edu

1 Department of Pharmaceutical Sciences, Northeast Ohio Medical University, 4209 State Route 44, Rootstown, OH 44272, USA

2 Department of Integrative Medical Sciences, Northeast Ohio Medical University, Rootstown, OH, USA
ATP adenosine triphosphate

CNS central nervous system

CTB cholera toxin-B subunit

D2 DBA/2J mouse

D2G DBA/2J-Gpnmb ${ }^{+}$

DMEM Dulbecco's modified essential medium

ECAR extracellular acidification rate

FC fluorocitrate

FCCP carbonyl cyanide

4-(trifluoromethoxy)phenylhydrazone

GLUT1 glucose transporter 1

IOP intraocular pressure

MCT2 monocarboxylate transporter 2

OCR oxygen consumption rate

ON optic nerve

PBS phosphate buffered saline

RBPMS RNA binding protein, multiple splicing

RGC retinal ganglion cell

TCA tricarboxylic acid. 


\section{Introduction}

Neurons, astrocytes, and blood vessels form a metabolic unit in the CNS. Glucose, lactate, and other metabolic intermediates obtained from the circulation are provided to neurons primarily through astrocytes, and enable glycolysis or oxidative phosphorylation to generate ATP. Neurotransmitters released from neurons can bind metabotropic glutamate receptors on astrocytes that increase intracellular calcium concentration, leading to generation of prostaglandins from arachidonic acid in astrocytes, and ultimately vasodilation to enable increased glucose uptake [1]. The glutamate-glutamine cycle, the astrocytic uptake of glutamate, and release of glutamine for neuronal uptake, can also provide carbons for the TCA cycle. These interactions not only enable flexibility but also engender dependence among the cells of the metabolic unit. Our understanding of these interactions and how they are altered by aging or disease are essential to our ability to manage neurodegenerative disease.

Evidence is emerging to support the critical role of energy management in axon degeneration observed in neurodegenerative disease, including glaucoma [2]. The glial-specific glucose transporter GLUT1 and the neuronal-specific monocarboxylate transporter MCT2 were significantly decreased in optic nerve prior to glaucoma-related degeneration [3]. Astrocytes compromised in their uptake of glucose paired with axons incapable of transporting lactate for fuel would preclude function unless the axons could obtain glucose directly. Neurons are capable of taking up glucose [4], but whether they can do so in a way that would sustain them under such conditions remains to be determined.

The DBA/2J mouse, a widely used model of glaucoma, undergoes a progressive optic neuropathy that results in asynchronous retinal ganglion cell death commencing between 10 and 12 months of age [5]. Deficits in physiological signaling prior to axon transport loss or axon degeneration was observed in the DBA/2J model of glaucoma [6]. Investigation into the cause of the signaling deficit suggested two sourcesthe mitochondria or the ways by which mitochondria obtain their energy substrate. We determined that the latter contributes to glaucomatous optic neuropathy by showing that critical glucose and monocarboxylate transporters are decreased prior to optic nerve degeneration [3]. The mitochondria, however, also show signs of compromise. Significantly lower mitochondria volume per volume of axon exists in glaucomatous optic nerve [7]. With intraocular pressure elevation, mitochondria in optic nerve of DBA/2J mice exhibited increased fission [8], and mitochondrial cristae loss [9]. It is likely these fragmented mitochondria have inefficient or dysfunctional oxidative phosphorylation, or increased reactive oxygen species production, potentially compromising the high metabolic demand of axons. In support of this, efforts to alter the energy balance (ketogenic diet, vitamin supplementation) toward greater substrate or cofactor availability to support oxidative phosphorylation have demonstrated significant improvement in retinal ganglion cell survival and function [3, 10]. Hence, there are large gains in neural function made possible with providing mitochondria with energy substrate.

An outstanding question is the nature of the axonal mitochondria deficit in the optic nerve. It is not possible to isolate axonal mitochondria from the optic nerve $(\mathrm{ON})$. Therefore, it is necessary to try to physiologically isolate axonal mitochondria and determine their function in situ. This investigation is the first to analyze mitochondrial function in axons using the Seahorse XFe24 Analyzer. Though not designed for tissue, the Seahorse XFe Analyzer has been used to measure oxygen consumption rate in sections or explants of rat and mouse brain $[11,12]$ and mouse retina [13]; however, no one has yet utilized it to investigate glaucomatous ON. Therefore, we decided to use this method to analyze individual glaucomatous ONs and answer a fundamental question: Whether and how mitochondrial respiration is altered with glaucoma progression. Given that the ON contains mitochondria from axons and glial cells, we would use the glial-specific aconitase inhibitor fluorocitrate to isolate the contribution of axonal mitochondria. Astrocytes and oligodendrocytes that take up fluorocitrate suspend oxidative phosphorylation due to inhibition of the TCA cycle [14]. These methods have shown us that glaucoma ON is energy hungry, with signs of not only increased glycolysis but also oxidative phosphorylation. Despite significant oxygen consumption, glaucoma ON has low maximal respiration. Critically, the glaucomatous ON loses its capacity for metabolic switching - it cannot respond to $\mathrm{F}_{0} \mathrm{~F}_{1}$-ATPase inhibition with increased glycolysis.

\section{Materials and Methods}

\section{Mice}

The DBA/2J (D2) mouse, an inbred strain that develops increased intraocular pressure (IOP) secondary to an iris pigment dispersion disease $[15,16]$, and its control strain, the DBA/2-Gpnmb ${ }^{+}$(D2G), were used for all experiments. Mice were obtained from Jackson Laboratories, Bar Harbor, ME. The D2 is a model of secondary glaucoma that recapitulates key features of pathology observed in human patients with primary open angle glaucoma $[5,15,17,18]$. The D2G control mice share the D2 background but carry a wildtype allele for the gpnmb gene.

$\mathrm{D} 2$ and $\mathrm{D} 2 \mathrm{G}$ mice at 3, 6, and 10 months of age were used for each of two experiments: one with and one without fluorocitrate treatment. For the non-fluorocitrate experiment, 10,14 , and 40 mice each of the D2 and D2G strains at 3,6, and 10 months of age, respectively, were used. For the fluorocitrate experiments, 10, 10, and 40 each of the D2 and 
D2G strains at 3, 6, and 10 months of age, respectively, were used. Mice were bred and housed on a $12 \mathrm{~h}$ light/dark cycle with access to standard rodent chow and water ad libitum. All procedures abide by the Statement for the Use of Animals in Ophthalmic and Vision Research and were approved by the Northeast Ohio Medical University Institutional Animal Care Committee.

\section{Visual Acuity Testing}

Visual acuities were established in mice using a forced-choice swim behavioral task consisting of 2 sessions of 10 trials per day over a period of approximately 4 weeks. The visual discrimination task apparatus (Cerebral Mechanics) was developed by Prusky [19, 20]. It uses a vertical sinusoidal grating to detect visual perceptual threshold by tasking mice to swim toward the visual stimulus they have associated with a submerged platform. Mouse visual acuity thresholds were determined by systematically increasing or decreasing the grating until animals consistently ( $\geq 3$ sessions) found the platform without error $\geq 7$ out of 10 trials per session while failing the next higher spatial frequency for at least 3 sessions. This threshold was recorded as their visual acuity. D2 mice have performed this task successfully at the full range of age and glaucoma-related pathology [21, 22].

\section{Anterograde Axonal Transport}

Mice were anesthetized with isoflurane (2.5\%) 3 days prior to sacrifice and $1.5 \mu \mathrm{l}$ of cholera toxin-B conjugated to AlexaFluor-488 (CTB-488; ThermoFisher Scientific) was injected into the posterior chamber of the eye. After mice were euthanized with an overdose of $390 \mathrm{mg} / \mathrm{mL}$ sodium pentobarbital (Beuthanasia-D), brains were dissected out, post-fixed, cryoprotected, then sectioned. Every sixth section was mounted on slides, coverslipped, and imaged at $\times 50$ magnification using an AxioZoom stereomicroscope (Zeiss). An ImageJ-FIJI (Schindelin et al. 2012) macro, available by request, was used to calculate the percentage of the area of the retinorecipient portion of the superior colliculus (percent area fraction) showing fluorescent labeling from the CTB conjugate compared to background labeling as evident in the deep layers of superior colliculus [23]. Data are presented as percent area fraction.

\section{Intraocular Pressure}

Terminal intraocular pressure measurements were taken prior to intraocular cholera toxin-B injections. Mice were lightly anesthetized using inhaled isoflurane $(2.5 \%)$ and ten consecutive IOP measurements were taken using a TonoLab (Tiolat Oy, Vantaa, Finland) rebound tonometer.

\section{Seahorse Bioanalyzer}

Oxygen consumption rates were measured in acutely isolated optic nerve using a Seahorse XF24 extracellular flux analyzer (Agilent). This plate-based system measures oxygen and proton release using fiber optic sensors that report oxygen consumption rate as well as extracellular acidification rate, representing measures of oxidative phosphorylation and glycolysis, respectively. Using fresh tissue in the Seahorse XF24 required optimization of media and compound concentrations, described below, as well as 40 additional D2 and D2G mice. All compounds and media were from Sigma-Aldrich unless otherwise specified.

Each Seahorse XF24 Sensor Cartridge plate was incubated in calibrant solution in a $37^{\circ} \mathrm{C} \mathrm{CO}_{2}$-free incubator 1 day prior to the experiment. Optic nerves (ONs) were carefully dissected from the base of the skull after removing the globe with a scalpel blade, then placed in $37{ }^{\circ} \mathrm{C}$ warmed DMEM supplemented with $25 \mathrm{mM}$ glucose, $4 \mathrm{mM}$ glutamine, and $0.5 \mathrm{mM}$ sodium pyruvate (buffering capacity $9.45 \times 10^{-4} \pm 1.13 \times$ $10^{-4} \mathrm{M}$ ). These high substrate concentrations were initially necessary to keep the ONs respiring through the extended assay time. However, for the fluorocitrate experiments, we empirically determined it was feasible to use a DMEM base media with $2 \mathrm{mM}$ glucose, $4 \mathrm{mM}$ glutamine, and $0.5 \mathrm{mM}$ sodium pyruvate (DMEM-low-glucose). Once all ONs were collected for the run, they were removed from DMEM then chopped into $500 \mu \mathrm{m}$ thick pieces using a Mclllwain tissue chopper. Pieces were secured to the mesh insert of a Seahorse XF24 Islet Capture Microplate within a clot made from $5 \mu \mathrm{L}$ of chicken plasma (Cocalico Biologicals) and 2-5 $\mu \mathrm{L}$ of bovine thrombin. One full ON was placed on each insert (see Fig. 1) then inverted and secured in the microplate well that contained warmed, supplemented DMEM.

Ports were loaded with $75 \mu \mathrm{L}$ of compounds in solution across the four ports that feed into each well of the sensor cartridge: Port A contained oligomycin A at $10 \times$ the final concentration $(100 \mu \mathrm{g} / \mathrm{ml})$; Port B held carbonyl cyanide 4-(trifluoromethoxy)phenylhydrazone (FCCP) $(44 \mu \mathrm{M})$ and sodium pyruvate $(110 \mathrm{mM})$ at $11 \times$ final concentration; Port $\mathrm{C}$ was media without metabolic substrates; and Port D held antimycin $\mathrm{A}$ at $13 \times$ the final concentration $(130 \mu \mathrm{M})$. In the fluorocitrate experiments, Port A contained fluorocitrate at $10 \times$ the final concentration $(2500 \mu \mathrm{M})$; Port B contained oligomycin A $(110 \mu \mathrm{g} / \mathrm{ml})$ plus either glucose or 2deoxyglucose $(88 \mathrm{mM})$, both at $11 \times$ the final concentration; Port $\mathrm{C}$ contained FCCP and sodium pyruvate at $12 \times$ final concentration $(48 \mu \mathrm{M}$ and $120 \mathrm{mM}$, respectively); Port D was loaded with antimycin A at $13 \times$ final concentration $(130 \mu \mathrm{M})$. The sensor cartridge was placed into the Seahorse XF24 to run an automated calibration and equilibration.

The compounds are fed from the ports into each well in sequence (A through D). Oligomycin A inhibits $F_{1} F_{0}$-ATP 
Fig. 1 Experimental design. The initial, non-fluorocitrate experiments took place as shown, with mice first placed in the forced-choice swim task in order to establish their visual acuity. Mouse intraocular pressure (IOP) was measured, then mice received bilateral posterior chamber injections of cholera toxin-B conjugated to AlexaFluor-488 (CTB-488). Three days later, mice were sacrificed and optic nerves (ONs) were chopped, secured within a fibrin clot and inverted into a Seahorse Islet Capture plate for analysis. Retinas were dissected, fixed, and immunolabeled with RBPMS. Brains were fixed, sectioned, and analyzed for CTB-488 quantity in the superior colliculus (SC). See "Materials and Methods" for additional detail. The fluorocitrate experiments included IOP measurement and RGC immunolabeling, but did not include CTB-488 injection, nor visual acuity determination
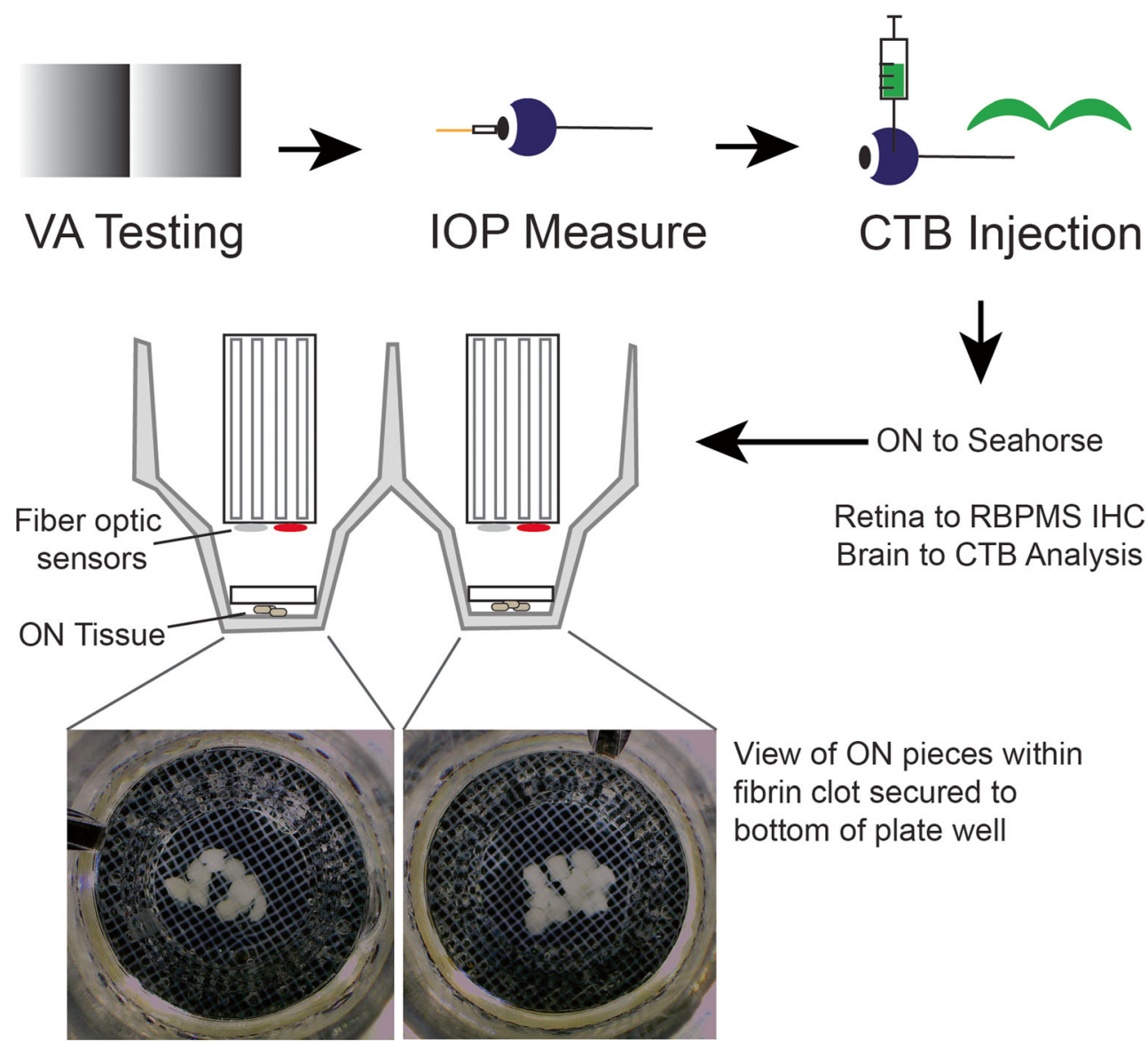

synthase, the enzyme responsible for synthesizing ATP from ADP and a phosphate, thereby decreasing oxygen consumption rate (OCR). FCCP is a mitochondrial uncoupler that allows proton movement across the mitochondrial membrane; its effect is to force the complexes of the electron transport chain to work to maintain the electrochemical gradient, thereby maximizing oxygen consumption. In our preparations, sodium pyruvate was added with the FCCP to further boost mitochondrial respiration. Antimycin A is a complex III inhibitor, the addition of which shuts down mitochondrial respiration. Fluorocitrate is a selective inhibitor of glial cell mitochondrial respiration [24]. Fluorocitrate was prepared as described [25]. Briefly, $8 \mathrm{mg}$ of DL-fluorocitric acid barium salt was added to $1 \mathrm{ml}$ of $0.1 \mathrm{M} \mathrm{HCl}$. Three drops of $0.1 \mathrm{M}$ $\mathrm{Na}_{2} \mathrm{SO}_{4}$ were added to precipitate the salt, followed by $2 \mathrm{ml}$ of $\mathrm{Na}_{2} \mathrm{HPO}_{4}$. The solution was centrifuged at $1000 \times \mathrm{g}$ for $5 \mathrm{~min}$. The supernatant was removed and $\mathrm{pH}$ adjusted to 7.4. Fluorocitrate was used at a final concentration of $250 \mu \mathrm{M}[26,27]$. Glucose and 2-deoxyglucose (2-DG) were used as a substrate and non-metabolizable glucose analog, respectively, in the fluorocitrate experiments. OCR values under specific conditions set by injection of each of these compounds can be used to calculate basal respiration, ATP-linked respiration, maximal respiration, spare capacity, and non- mitochondrial respiration. Table 1 shows the calculations behind values expressed in the figures that document OCRs.

We undertook several optimization experiments to determine the mix, waiting, and measurement protocols to assess oxygen consumption rate. See Table 2 for the protocol used for experiment 1, without fluorocitrate, and experiment 2, with fluorocitrate. The entire time within-instrument for the ONs was $4 \mathrm{~h} 45 \mathrm{~min}$.

\section{Data Processing}

For each baseline OCR, the mean of the last three measurements prior to the first injection was used to represent the baseline OCR. For the oligomycin A response, the minimum OCR value obtained was used, as was the maximal value obtained after FCCP injection. For antimycin A OCR, the mean of the three values after the minimum were used. Wells that did not attain at least a baseline uncorrected OCR of 50 pmol $\mathrm{O}_{2} /$ min were not used in subsequent analyses; in addition, wells were rejected if the ECAR values were negative.

Following the Seahorse XF24 runs, optic nerves were removed from the mesh inserts and sonicated (2-4 3-s pulses at $10 \%$ amplitude) using a Branson Sonifier in $50 \mu \mathrm{l}$ of protein 
Table 1 Respiration indices and their means of calculation

\begin{tabular}{ll}
\hline Index & Calculation of index value \\
\hline Basal respiration & Baseline OCR - non-mitochondrial respiration \\
ATP production & Basal respiration-oligomycin OCR \\
Maximal respiration & FCCP respiration-non-mitochondrial respiration \\
Spare capacity & FCCP respiration-basal respiration \\
Non-mitochondrial respiration & OCR with antimycin A treatment \\
Coupling efficiency & ATP production/basal respiration \\
\hline
\end{tabular}

$O C R$, oxygen consumption rate

lysis buffer with protease and phosphatase inhibitors followed by BCA assay (Pierce) to determine total protein concentration. OCR and ECAR data was corrected for the amount of ON protein in each well. OCR data was also normalized to baseline.

For the fluorocitrate experiments, data were normalized to the baseline OCR obtained in the presence of fluorocitrate. Fluorocitrate had a significant effect on OCR, so all subsequent data would be expressed in terms of the fluorocitrate baseline OCR. The data are expressed in graphs as \% fluorocitrate OCR.

\section{Immunohistochemistry}

Freshly isolated eyes were immersion fixed in $4 \%$ paraformaldehyde for $30 \mathrm{~min}$ then cryoprotected in $30 \%$ sucrose with $0.02 \%$ sodium azide. Retinas were dissected out and vitreous removed. Immunolabeling included washes in $0.1 \mathrm{M}$ PBS,

Table 2 Seahorse XF24 protocol

\begin{tabular}{|c|c|c|c|c|c|}
\hline \multicolumn{4}{|c|}{ Calibration and equilibration } & \multirow[t]{2}{*}{$\sim 30 \mathrm{~min}$} & \multirow{2}{*}{$\begin{array}{l}\text { Calibrate } \\
\text { Equilibrate }\end{array}$} \\
\hline & & & & & \\
\hline \multirow[t]{3}{*}{ Baseline } & & & & $1 \times$ & $\operatorname{Mix}(1.5 \mathrm{~min})$ \\
\hline & & & & $4 \times$ & Wait (2.0 min) \\
\hline & & & & & Measure $(3.0 \mathrm{~min})$ \\
\hline \multirow{24}{*}{$\begin{array}{l}\text { Experiment 1: no } \\
\quad \text { fluorocitrate }\end{array}$} & Oligomycin & Experiment 2: fluorocitrate & Fluorocitrate & & Inject port $\mathrm{A}$ \\
\hline & & & & $1 \times$ & $\operatorname{Mix}(1.5 \mathrm{~min})$ \\
\hline & & & & $3 \times$ & Wait (10.0 min) \\
\hline & & & & & Measure $(3.0 \mathrm{~min})$ \\
\hline & & & & $3 \times$ & Wait (2.0 min) \\
\hline & & & & & Measure $(3.0 \mathrm{~min})$ \\
\hline & FCCP and sodium pyruvate & & Oligomycin and 2-DG or glucose & & Inject port B \\
\hline & & & & $1 \times$ & $\operatorname{Mix}(1.5 \mathrm{~min})$ \\
\hline & & & & $3 \times$ & Wait (10.0 min) \\
\hline & & & & & Measure $(3.0 \mathrm{~min})$ \\
\hline & & & & $3 \times$ & Wait (2.0 min) \\
\hline & & & & & Measure $(3.0 \mathrm{~min})$ \\
\hline & DMEM w/o Glc, sodium pyruvate & & FCCP and sodium pyruvate & & Inject port $\mathrm{C}$ \\
\hline & & & & $1 \times$ & $\operatorname{Mix}(1.5 \mathrm{~min})$ \\
\hline & & & & $3 \times$ & Wait (10.0 min) \\
\hline & & & & & Measure $(3.0 \mathrm{~min})$ \\
\hline & & & & $3 \times$ & Wait (2.0 min) \\
\hline & & & & & Measure $(3.0 \mathrm{~min})$ \\
\hline & Antimycin A & & Antimycin A & & Inject port $\mathrm{D}$ \\
\hline & & & & $1 \times$ & $\operatorname{Mix}(1.5 \mathrm{~min})$ \\
\hline & & & & $2 \times$ & Wait (12.0 min) \\
\hline & & & & & Measure (3.0 min) \\
\hline & & & & $7 \times$ & Wait (2.0 min) \\
\hline & & & & & Measure $(3.0 \mathrm{~min})$ \\
\hline
\end{tabular}


then incubation in blocking solution (5\% donkey serum, $1 \%$ Triton X-100 in $0.1 \mathrm{M}$ PBS) for $1 \mathrm{~h}$, primary antibody (diluted in $0.5 \%$ bovine serum albumin, $0.9 \% \mathrm{NaCl}, 1 \%$ Triton $\mathrm{X}-100$ in 0.1 M PBS) for 48-72 h, washes in 0.1 M PBS, blocking solution for $30 \mathrm{~min}$, secondary antibody incubation for $18 \mathrm{~h}$, washes, DAPI labeling, then coverslipping with Fluoromount-G. RBPMS (RNA binding protein, multiple splicing) primary antibody (Genetex, Irvine, CA) to label RGCs (1:250) and secondary antibody from Jackson ImmunoResearch (West Grove, PA) Alexa Fluor 594conjugated anti-rabbit IgG (1:250) were used. Images were collected on a Leica DMi8 confocal microscope integrated with Leica application Suite X 3.1.1.15751 (Leica Microsystems, Buffalo Grove, IL, USA). Figures were created using Adobe Illustrator, Adobe Creative Cloud version 2017.

\section{Stereological Analysis}

RBPMS-immunolabeled retinal ganglion cells were quantified in whole mounted retinas by unbiased stereology using StereoInvestigator (MBF Bioscience, VT), a $\times 40$ objective, and the optical fractionator approach. Between 35 and 40 sampling sites $(10 \%)$ of each retina were counted. The coefficient of error (Schmitz-Hof) was maintained at 0.05 or below, ensuring sufficient sampling rate.

\section{Statistics}

Power analysis to determine appropriate sample number was undertaken using GPower 3.1 software. For $80 \%$ power and using the standard deviation of OCR, we would need 10 optic nerves per strain per time point. For these experiments, we used at minimum 10 optic nerves, but usually 20 to 40 per time point, especially at 10 months of age. Statistical analyses were performed with GraphPad Prism software version 7.0. Figure graphs were made using GraphPad Prism 7.0. Unpaired, two-tailed $t$ tests were used when comparing across groups within a strain, or within a group across strains. For not normally distributed data, non-parametric tests were employed. One-way ANOVA and Tukey's multiple comparison post hoc test was used when comparing across multiple groups within a strain, or across multiple groups within an outcome measure. $p<0.05$ was considered significant, and data are reported as mean $\pm \mathrm{SD}$.

\section{Results}

Optic nerves from D2G (control) and D2 (glaucoma) mice were removed and processed as described in Fig. 1. Intraocular pressure (IOP) measured in all $\mathrm{D} 2$ and $\mathrm{D} 2 \mathrm{G}$ mice at 3, 6, and 10 months of age showed a significant increase in IOP across age in the D2, but no change in the D2G (Fig. 2a).
IOP in 10-month-old D2 mice was significantly higher than at 6 months, and IOP at 6 months was significantly higher than at 3 months. IOP increase is accompanied by retinal ganglion cell (RGC) loss in the D2 mice. Figure $2 b$ shows the range of RGC density for retinas taken from the mice used in the nonfluorocitrate experiments. RGCs were immunolabeled with RBPMS, an RGC-specific marker, then quantified using unbiased stereology. There is a significant decrease in RGC number from 6 to 10 months of age in the D2 mice; RGC numbers at 6 and 10 months in the D2 are also significantly lower than RGC density in 6- and 10-month-old D2G mice (Fig. 2b). An expectation of vision loss with RGC loss was observed in the D2 mice. Visual acuity in the mice for the nonfluorocitrate experiments was measured using a forced-choice swim task in which the mice find a hidden platform they have been trained to associate with a visual stimulus [22]. The visual acuity in the D2 mice decreased significantly with aging (Fig. 2c). Visual acuity at 10 months of age in the D2 was significantly lower than that of 10-month-old D2G mice. There was also a significant decline in visual acuity in the D2G from 3 to 6 months of age (Fig. 2c). An additional measure of pathology in the $\mathrm{D} 2$ model of glaucoma is anterograde axon transport deficit, as measured in the $\mathrm{D} 2$ and $\mathrm{D} 2 \mathrm{G}$ mice by quantifying the percent of the superior colliculus (SC) area positive for cholera toxin-B conjugated to AlexaFluor-488 (Fig. 2d). Reduced CTB in the SC indicates a deficit in the RGC transport of CTB from the retina to the retinorecipient regions in the brain $[23,28]$. There was a significant decrease in $\mathrm{CTB}+$ area of the $\mathrm{SC}$ with age in the D2 mice (Fig. 2d). There was also a significant decrease in the CTB labeling of the 10-month-old D2 versus the 10-month-old D2G SC. Examples of RGC distribution in flatmount retina from $\mathrm{D} 2 \mathrm{G}$ and D2 10-month-old mouse eyes are shown in Fig. 2e, with high-magnification insets. Figure $2 \mathrm{f}$ depicts a typical CTB labeling result in the D2G and D2 SC.

\section{Oxygen Consumption Rate in Optic Nerve}

Optic nerves from the mice whose retinas and SC were analyzed as described above were removed and used for the Seahorse XF Analyzer experiments that were designed to evaluate mitochondrial function by measuring oxygen consumption rate through the sequential addition of inhibitors of oxidative phosphorylation. The average oxygen consumption rate (OCR) for 3-month-old D2G and D2 ON is shown in Fig. 3a. These data represent the respiration for all mitochondria within all of the cell types (astrocytes, oligodendrocytes, microglia, endothelial cells) and axons in the ON. Initially, data were corrected for the amount of protein within the well (after the Seahorse run, ONs were removed and concentrations measured) then normalized to baseline OCR. Comparisons of OCR after oligomycin, FCCP, or antimycin 

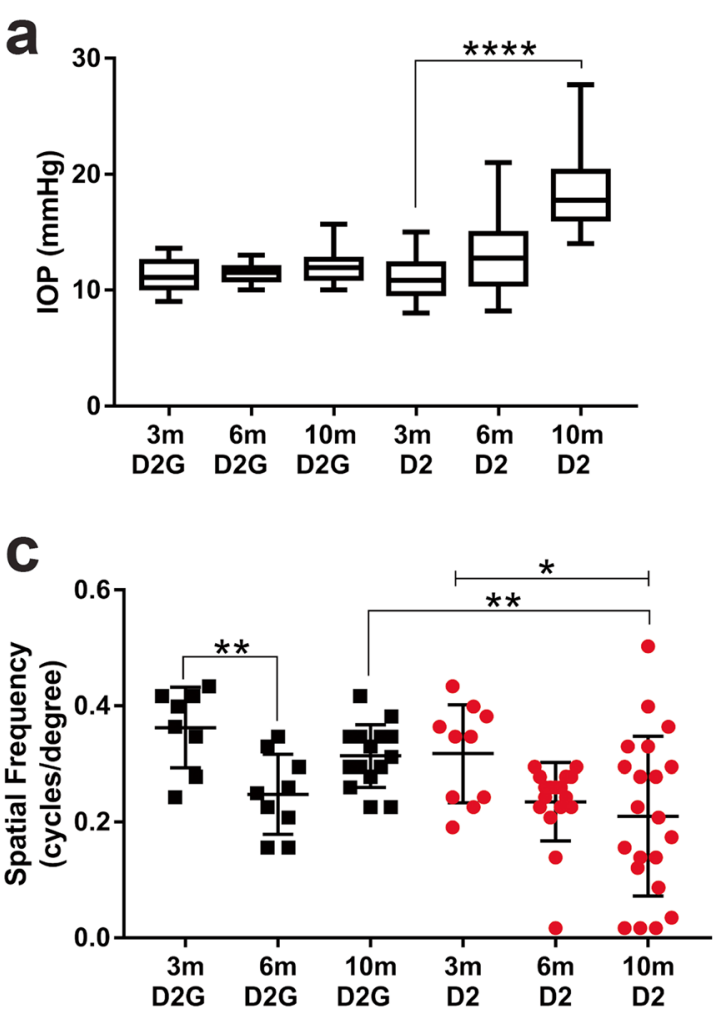

e
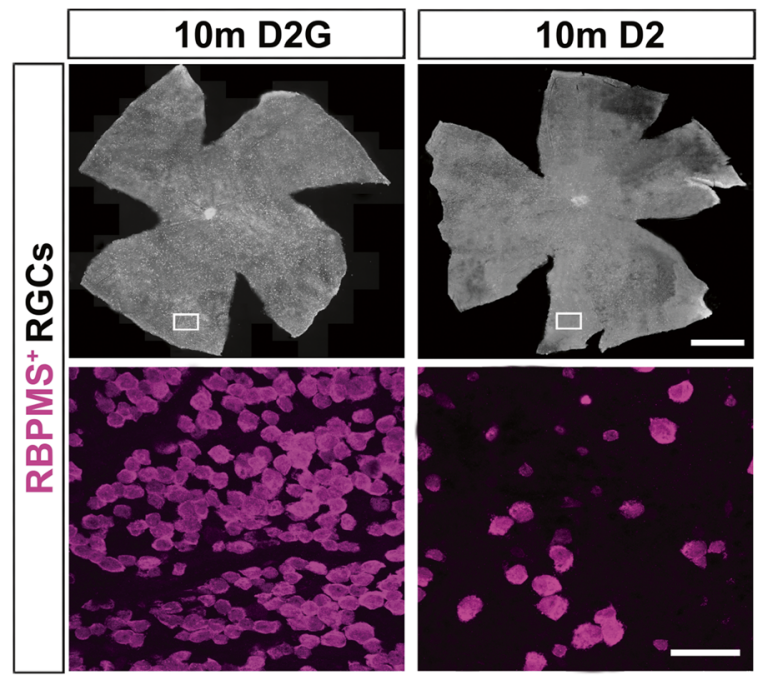

Fig. $2 \mathrm{DBA} / 2 \mathrm{~J}$ retina and brain show degenerative changes by 10 months of age. a Intraocular pressure (IOP) measured in all 3-, 6-, and 10-monthold DBA/2J (D2) and DBA/2J-Gpnmb ${ }^{+}$(D2G) indicates a significant IOP increase by ANOVA (Kruskal-Wallis $H$ test, $\chi^{2}(2)=54.19$, $* * * * p<0.0001)$ in the $\mathrm{D} 2$ mice from 3 to 10 months. There was no change in average IOP across the $\mathrm{D} 2 \mathrm{G}$ mice. The increases from 3 to 6 months, and 6 to 10 months in the D2 were statistically significant $(t$ test, $p=0.0091$ and $p<0.0001$, respectively). b-d Outcome measures for mice in the non-fluorocitrate experiments. b Retinal ganglion cell (RGC) density in the D2 and D2G mice at 3, 6, and 10 months of age indicates significantly decreased RGC density in 6 month D2 versus D2G $(t$ test, $* p=0.0482)$ and in 10 -month D2 versus D2G $(t$ test, $* * * p=0.0005)$. The 10 -month-old D2 retina also had significantly lower RGC density than 6month-old D2 retina ( $t$ test, $* * * * p<0.0001)$. $\mathbf{c}$ Visual acuity of mice as spatial frequency threshold in cycles/degree shows a significant decline in visual acuity in the D2 mice from 3 to 10 months of age (ANOVA,
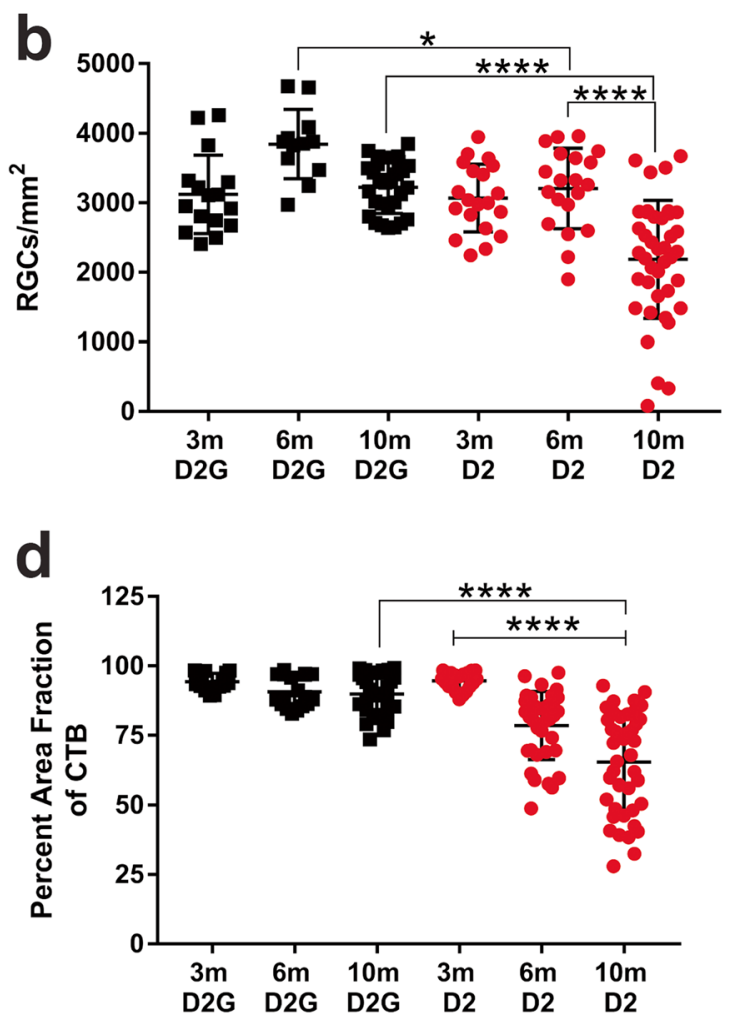

f
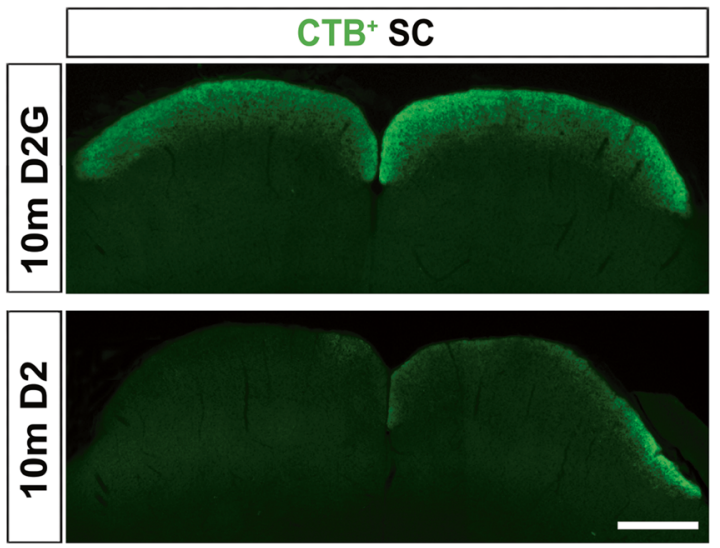

$\left.F_{2,45}=3.482, * p=0.0393\right)$. There was a significant decline in the D2G mice from 3 to 6 months of age ( $t$ test, $* * p=0.0039)$. The visual acuity was also significantly lower in the 10 -month-old D2 versus D2G mice $(t$ test, $* * p=0.0091)$. d The percent area fraction of cholera toxin-B (CTB) fluorescent label in the superior colliculus (SC) showed a significant decline with aging and pathology in the D2 mice (ANOVA, $F_{2,95}=$ $29.95, * * * * p<0.0001)$. There was also a significant difference in CTB label in the 10-month-old D2 versus D2G mice ( $t$ test, $* * * * p<0.0001)$. e Photomicrographs of flatmount retina from 10-month-old D2G and D2 mice. Top panels show flatmount retina with white squares to identify the areas from which the high-magnification insets were drawn. Scale bar = $500 \mu \mathrm{m}$. Insets show RBPMS-positive RGCs (magenta) in corresponding flatmount retina. Scale bar $=50 \mu \mathrm{m}$. f Cross-sections of superior colliculus showing CTB labeling (green) in the retinorecipient portions of the superficial layers of D2G and D2 brain. Scale bar $=100 \mu \mathrm{m}$ 
A injections were not statistically different within age and across strain (Fig. 3a).

ATP production and other data derived from OCR values are calculated as depicted in Fig. 3b. Oxygen consumed for ATP production did not vary across ages in the $\mathrm{D} 2 \mathrm{G}$ mouse $\mathrm{ON}$. In the $\mathrm{D} 2$ mouse $\mathrm{ON}$, ATP production decreased significantly with age, Fig. 3c. ATP production was also significantly lower in the 6-month-old D2 ON when compared to 3 month D2 and the 6-month-old D2G $\mathrm{ON}$. The 10 month-old D2 ON oxygen consumption for ATP production did not differ from the 6- or 10-monthold D2G ON. Maximal respiration in the D2 and D2G ON decreased significantly with aging (Fig. 3d). Spare capacity, the potential for the system to accelerate ATP production, was significantly decreased with aging in the D2 but not the D2G ON (Fig. 3e). a

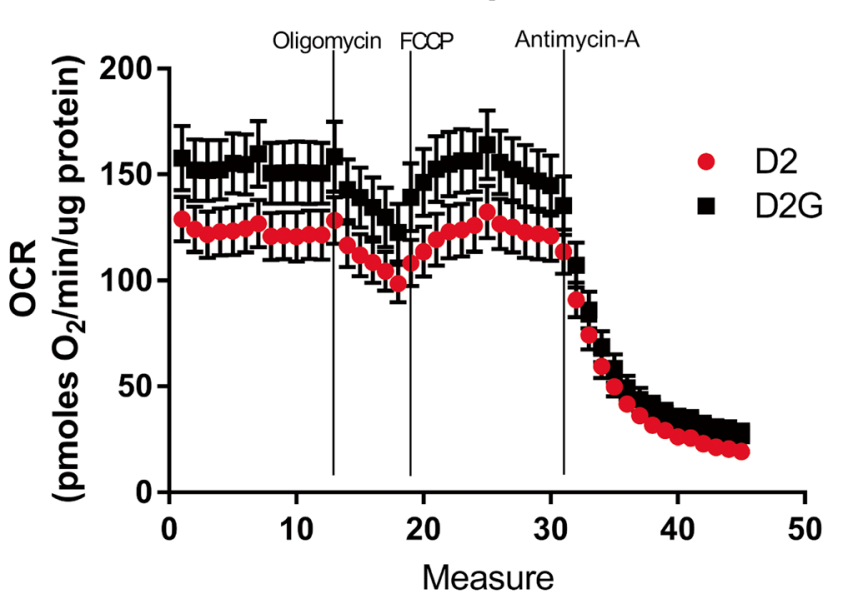

b

\section{Derivative Value Calculations}

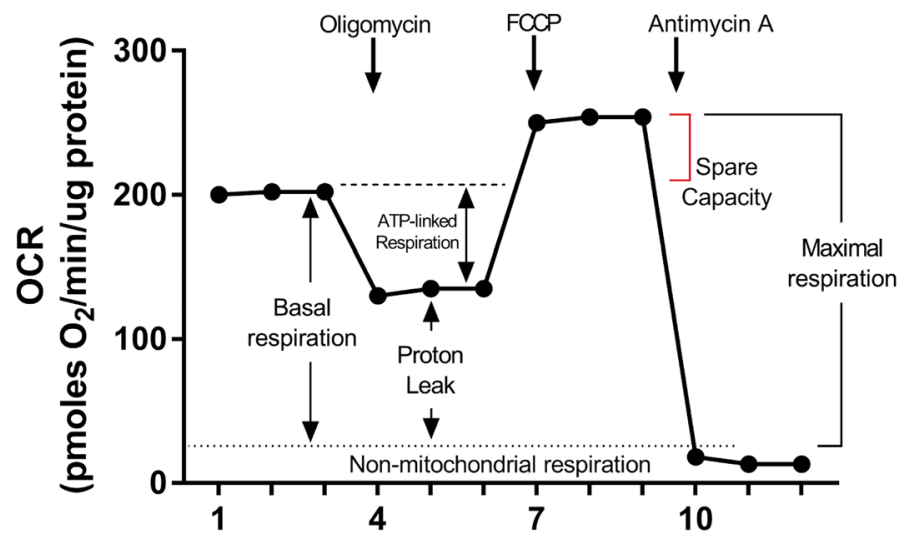

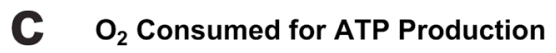
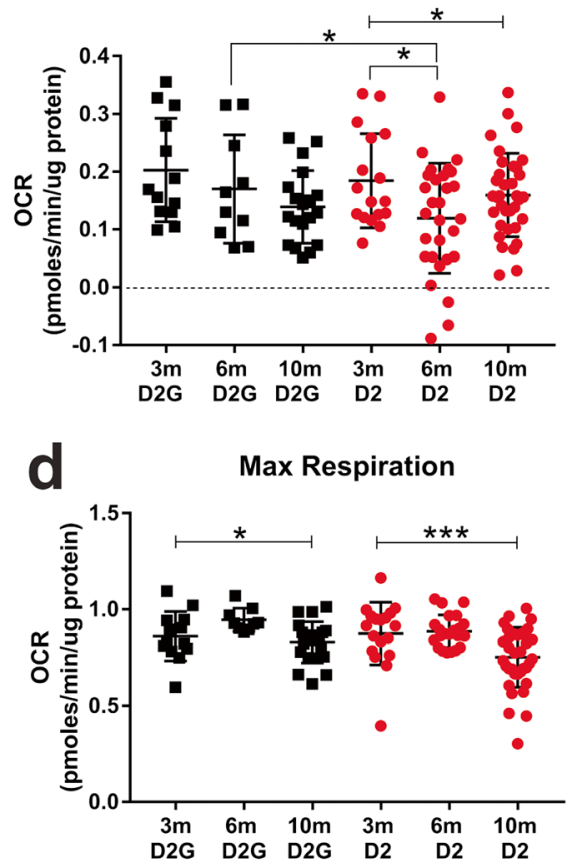

e

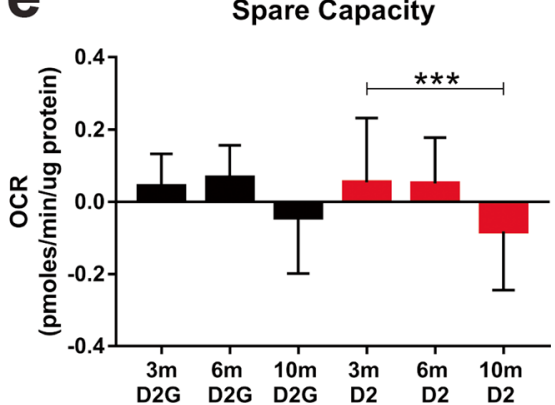

Fig. 3 Oxygen consumption rate (OCR) in D2 and D2G ON, normalized to amount of ON protein per well, used to calculate ATP production, maximal respiration, and spare capacity in $\mathrm{ON}$. Media for these experiments contained $25 \mathrm{mM}$ glucose, $4 \mathrm{mM}$ glutamine, and $0.5 \mathrm{mM}$ sodium pyruvate. a Mitochondrial respiration in the 3-month-old D2G ON (black squares), and the 3-month-old D2 ON (red circles). There were no statistical differences for OCR taken after oligomycin, FCCP, and antimycin A injections across strain within the 3,6, and 10-month-old age groups; only the 3-month-old graphs are shown as example output. OCR is $\mathrm{pmol} \mathrm{O}_{2} / \mathrm{min} / \mu \mathrm{g}$ protein $\pm \mathrm{SEM}$ for this graph only, to allow individual points to be visible. b Schematic showing the various calculated values for basal respiration (baseline), ATP-linked respiration (often referred to as ATP production), maximal respiration, and spare capacity, as derived from the oxygen consumption rate measured during a Seahorse Analyzer run. Arrows show the injection points for the oligomycin, the FCCP, and the antimycin A compounds that inhibit the $\mathrm{F}_{0} \mathrm{~F}_{1}$-ATPase, uncouple the mitochondrial membrane potential from ATP production, and inhibit Complex III of the electron transport chain, respectively. c ATP production decreased significantly with age in the D2 ON (ANOVA, $F_{2,78}=$ $3.632, * p=0.031)$. ATP production was significantly lower in the 6-monthold D2 ON when compared to 3-month D2 ( $t$ test, * $p=0.0236)$ and the 6month-old D2G ON ( $t$ test, $* p=0.032)$. ATP production did not vary across ages in the D2G mouse ON. d Maximal respiration in the D2 and D2G ON decreased significantly with aging (within strain for D2 Kruskal-Wallis $H$ test, $\chi^{2}(2)=14.78$, *** $p=0.0006$; for D2G, ANOVA, $F_{2,40}=3.771$, * $p=$ 0.0316). e Spare capacity, the potential for acceleration of ATP production when necessary, was significantly decreased with aging in the D2 but not the D2G ON (within strain for D2 Kruskal-Wallis H test, $\chi^{2}(2)=16.8$, *** $p=$ 0.0002 ; for D2G Kruskal-Wallis $H$ test, $\chi^{2}(2)=5.597, p=0.0609$ ) 


\section{Extracellular Acidification Rate Highest in Glaucoma Optic Nerve}

The extracellular acidification rate (ECAR) represents the release of protons into the extracellular milieu as a byproduct of glycolysis (from lactate production) but also the protons from substrate oxidation that are released with the export of $\mathrm{CO}_{2}$ that becomes hydrated to $\mathrm{H}_{2} \mathrm{CO}_{3}$ and dissociates to $\mathrm{HCO}_{3}{ }^{-}+$ $\mathrm{H}^{+}$[29]. Acidification from respiration compared to glycolysis varies widely by cell type [30]; however, as shown below in the fluorocitrate experiments, roughly half of the ECAR in D2G ON can be attributed to glycolytic activity. From the outset, the ECAR in the D2 ON was higher than in the D2G (Fig. 4a). At 6 and 10 months of age, the D2 ECAR was significantly greater than the D2G (Fig. 4a). These data suggest that glycolysis may provide a larger proportion of energy in the D2 ON than in the D2G, even in spite of the high glucose conditions (see "Materials and Methods"). In the presence of oligomycin, ECAR was significantly greater in the D2 ON compared to the D2G at both 6 months and 10 months of age (Fig. 4b). Since oligomycin inhibits the

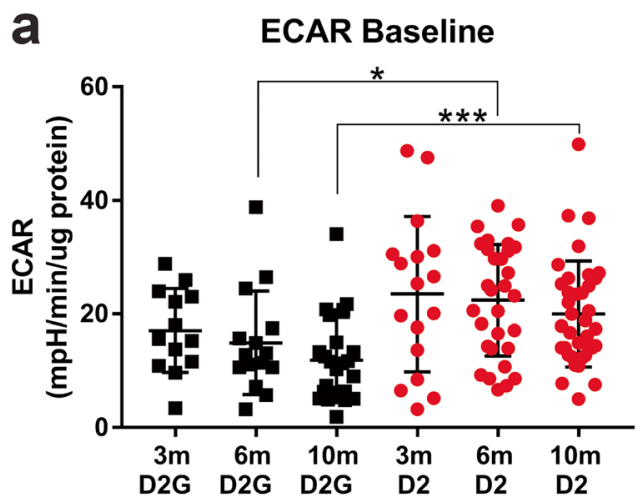

C

ECAR Difference

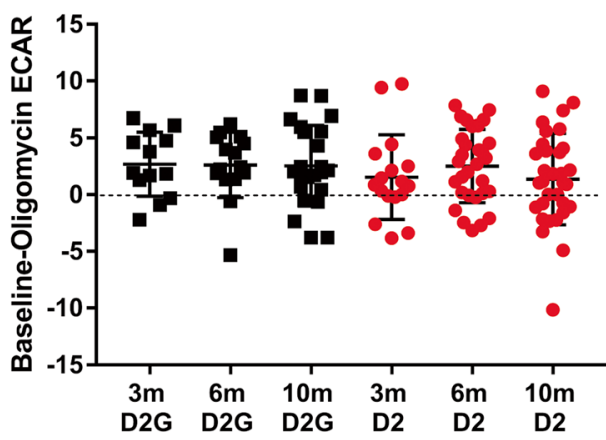

Fig. 4 Extracellular acidification rate (ECAR) normalized to protein per well in 3-, 6-, and 10-month-old D2 ON. a Baseline ECAR, normalized by amount of protein per well, was not statistically different in the D2 versus the $\mathrm{D} 2 \mathrm{G} \mathrm{ON}$ at 3 months of age ( $t$ test, $p=0.0564)$, but was significantly higher in the D2 ON versus the D2G at 6 months of age $(t$ test, $* p=0.013)$. Baseline ECAR was significantly higher in the 10 month-old D2 ON compared to the D2G ( $t$ test, *** $p=0.0003)$. b ECAR measured in the presence of oligomycin was not significantly
$\mathrm{F}_{0} \mathrm{~F}_{1}$-ATPase, the ECAR measures in the presence of oligomycin may increase over baseline ECAR, reflecting an increase in glycolysis to meet ATP needs. Both the D2 and D2G ON mean ECAR in the presence of oligomycin was increased over baseline ECAR (Fig. 4c), but there were individual ONs for each age and strain that did not show an increase in ECAR with oligomycin treatment.

The conditions of the first experiment supplied excess substrate $(0.5 \mathrm{mM}$ aerobic substrate sodium pyruvate and $25 \mathrm{mM}$ aerobic/anaerobic substrate glucose), a result of empirical optimization of conditions that would allow the ONs to respond through the 4+ hours of assay time. The OCR/ECAR ratio indicated the striking preference for glycolysis in the D2 ONs (Fig. 4d). Since the relatively high glucose concentration exceeds the physiological range, we endeavored to execute our fluorocitrate experiments (described below) using physiological glucose conditions $(2 \mathrm{mM}$ that rises by $8 \mathrm{mM}$ to $10 \mathrm{mM}$ with FCCP addition).

We also hypothesized that visual function (as measured by visual acuity), RGC number, anterograde axon transport, or IOP levels (Fig. 1) would predict changes in respiration

b

ECAR Oligomycin

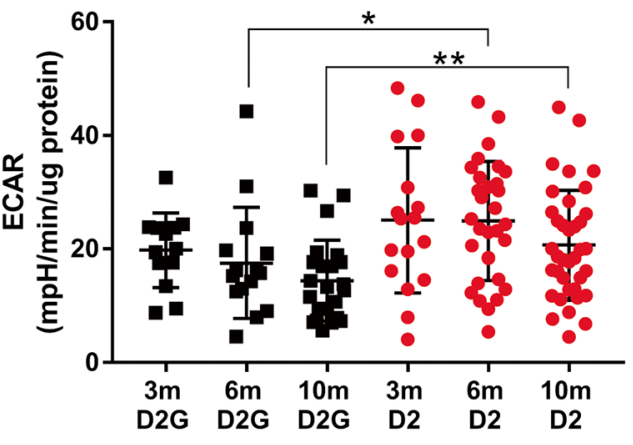

\section{0m Baseline OCR/ECAR}

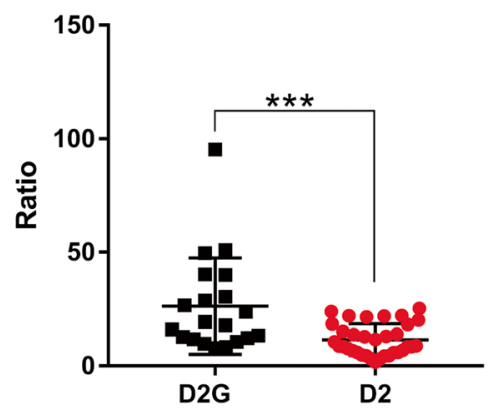

different in the D2 versus D2G ON at 3 months of age ( $t$ test, $p=$ 0.1863 ), but was significantly higher in 6-month-old D2 compared to D2G ON ( $t$ test, ${ }^{*} p=0.0282$ ). ECAR in the presence of oligomycin was significantly higher in the 10-month-old D2 compared to D2G ON ( $t$ test, $* * p=0.0099$ ). $\mathbf{c}$ The difference between ECAR at baseline and ECAR in the presence of oligomycin. $\mathbf{d}$ The ratio of OCR to ECAR in the ONs shows a significantly decreased ratio in the $\mathrm{D} 2 \mathrm{ON}(t$ test, $* * * p=$ 0.0006) 
indices. However, we determined this was not the case. For example, a plot of calculated maximal respiration against visual acuity for the D2 and D2G mice showed no difference in the slope of the regression line through the scatterplot, nor did a plot of basal respiration by RGC density (Online Resource Figure 1). ATP production, maximal respiration, or ECAR plotted by visual acuity binned into data ranges also showed no predictive power (Online Resource Figure 2). However, we did note that the higher the visual acuity, the greater oxygen consumption in the presence of FCCP for the D2 ON (Online Resource Figure $2 b$ ). Higher visual acuity suggests increased numbers of functional RGCs; these RGC axons show greater capacity for increased oxygen consumption. Finally, the ECAR in the ON taken from mice with visual acuity at 0.2 cyc/deg. or higher was not different across the D2 and D2G strains, but was significantly higher for the D2 mice with low visual acuity ( 0 to $0.2 \mathrm{cyc} / \mathrm{deg}$ ), Online Resource Figure 2c. This suggests that mice with low visual acuity drive much of the significantly increased ECAR baseline at 10 months of age as shown in Fig. 4.

\section{Fluorocitrate Unmasks Axonal Mitochondria Function}

Fluorocitrate (FC) inhibits the TCA cycle enzyme aconitase in astrocytes because of its preferential uptake by astrocytes, and possibly oligodendrocytes [14, 25]. Incubating optic nerve with FC should better isolate the contribution of axonal mitochondria to the mitochondrial respiration as measured by the Seahorse Analyzer. We injected FC in advance of the other port injections during a subset of Seahorse runs for D2 and $\mathrm{D} 2 \mathrm{G} \mathrm{ON}$ at 3, 6, and 10 months of age; Online Resource Figure $3 \mathrm{c}$ confirms aconitase inhibition with the FC concentration used here. Figure 5a shows the distribution of RGC density for the mice used in the FC experiments. There was a significant decline in RGC number with aging in the D2 retina. RGC number in 10-month-old D2 was significantly lower than in D2G retina (Fig. 5a).

There was no difference in baseline OCR between the $\mathrm{D} 2$ and D2G 10-month-old ON when data were normalized to protein (Fig. 5b). However, when baseline OCR was shown as a percentage of the OCR in the presence of FC, the OCR was significantly higher in the 10-month-old D2 than D2G ON (Fig. 5c). This indicated that the glia, whose mitochondria were inhibited with FC treatment, were contributing more to OCR in the D2 than the D2G ON at 10 months of age. Baseline OCR did not differ for D2 and D2G mice at 3 and 6 months of age (data not shown). FC, by blocking glial mitochondria, establishes a baseline oxygen consumption that represents that obtained from axons within the $\mathrm{ON}$; therefore, subsequent data is normalized to the FC baseline OCR. Online Resource Figure $3 a$ and $b$ shows the OCR through each stage of the FC experiment for one example each of the D2 and $\mathrm{D} 2 \mathrm{G} \mathrm{ON}$ at 10 months of age. Oligomycin injection in the presence of FC resulted in significantly lower OCR in the D2 ON than the D2G (Fig. 5d). The magnitude of oligomycinassociated decrease in OCR reflects ATP-linked respiration, suggesting that D2 axonal mitochondria are producing more ATP than those in the D2G control strain. Figure 5e shows that the oxygen consumed for ATP production (derived from the difference between FC and oligomycin OCR) is significantly higher in $\mathrm{D} 2$ than $\mathrm{D} 2 \mathrm{G} \mathrm{ON}$ in the presence of $\mathrm{FC}$, suggesting the axonal mitochondria in the $\mathrm{D} 2 \mathrm{ON}$ are more productive than those in the D2G ON. Proton leak, the OCR attributable to proton movement across the inner mitochondrial membrane independent of $\mathrm{F}_{0} \mathrm{~F}_{1}$-ATPase activity, was not different between the D2 and D2G ON at 10 months (data not shown). In contrast to ATP production, maximal respiration as detected after FCCP injection in the presence of FC was significantly lower in the D2 ON compared to the D2G at 6 months of age (Fig. 5f) and at 10 months (Fig. 5g). Coupling efficiency, the fraction of basal respiration that drives ATP synthesis, is significantly higher in the 10-month D2 ON (Fig. 5h).

\section{ECAR in the Presence of Fluorocitrate Shows Limits to Glycolysis}

The 10-month D2 ON exhibited significantly higher baseline ECAR activity (release of protons) prior to $\mathrm{FC}$ injection than D2G ON (Fig. 6a). ECAR in the presence of FC did not change (Online Resource Figure 3d), suggesting that the ECAR in the ON does not appreciably comprise proton release from astrocytic respiration. ECAR was not different between D2 and D2G ON at 3 months nor at 6 months during oligomycin treatment in the presence of FC (data not shown). However, at 10 months of age, ECAR was significantly greater in the D2G ON compared to the D2 ON with oligomycin treatment in the presence of $\mathrm{FC}$, indicating that inhibition of the $\mathrm{F}_{0} \mathrm{~F}_{1}$-ATPase increased glycolysis (as shown with increased ECAR) in D2G ON to meet ATP need, but the D2 ON showed an impaired ability to meet this challenge (Fig. $6 b)$. We also measured ECAR from D2 and D2G ON that were incubated with 2-deoxyglucose (2-DG) instead of glucose. Without glucose, glycolysis should be hampered, and ECAR levels ascribable to glycolysis should also decrease. This occurred in both D2 and D2G ON, as shown in Fig. 6b. It is possible to estimate the ECAR that comes from substrate oxidation in both the $\mathrm{D} 2$ and $\mathrm{D} 2 \mathrm{G}$ by comparing the difference in ECAR with and without 2-DG. In the D2 ON, the decline in ECAR ratio with glucose versus 2-DG is $29 \%$. Therefore, $\sim 71 \%$ of the ECAR ratio in the $\mathrm{D} 2 \mathrm{ON}$ is likely from substrate oxidation-based proton release. For the D2G ON, the ECAR ratio decreased by $50 \%$ with $2-\mathrm{DG}$ compared to glucose, indicating about half of the ECAR ratio is from substrate oxidation. Collectively, these data indicate that having higher levels of ECAR in the 10-month D2 axons (Fig. 6a, b) is accompanied by an impairment in their ability to respond 

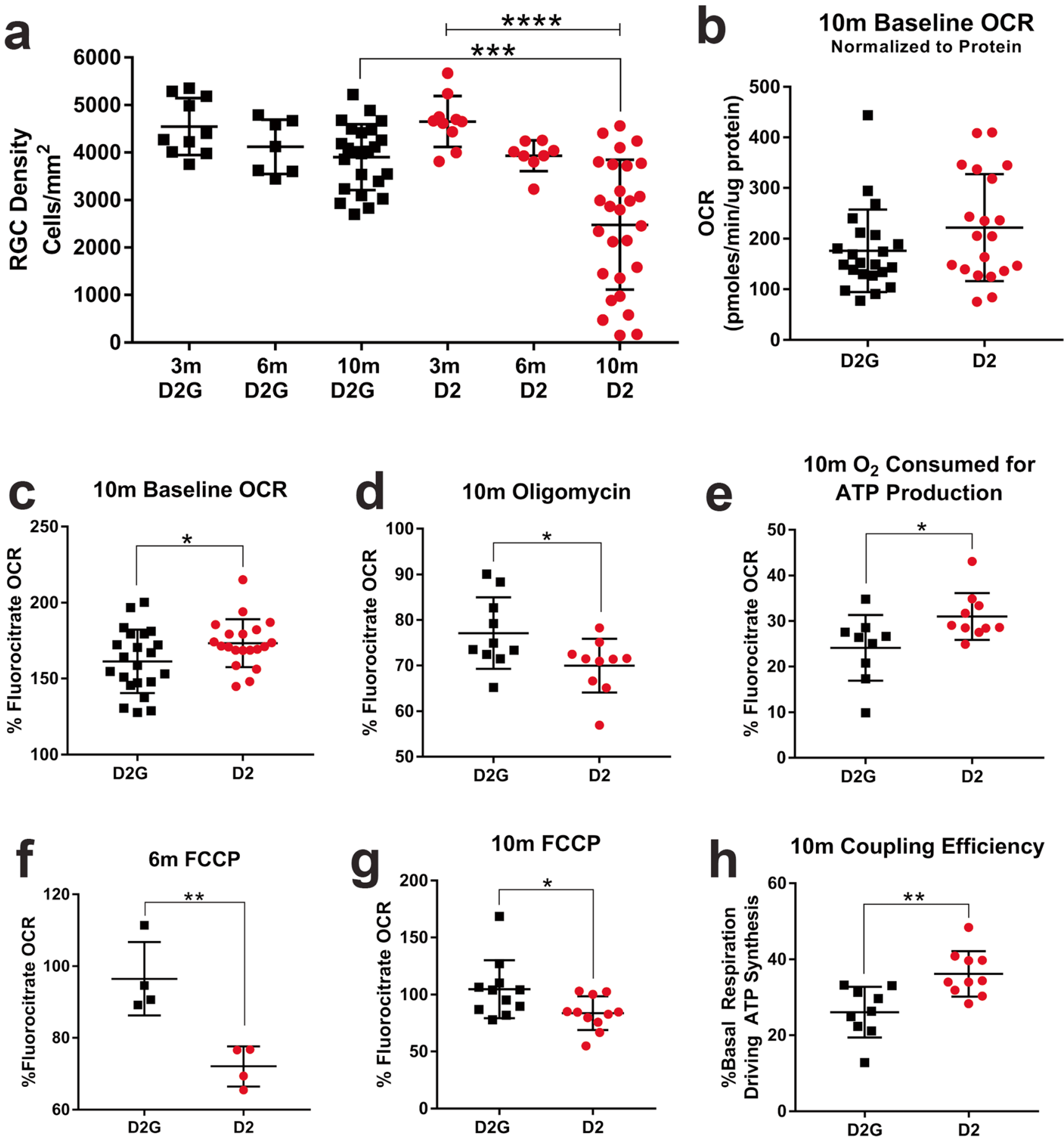

Fig. 5 RGC density and mitochondrial respiration for mice used in the fluorocitrate experiments. Media for these experiments contained $2 \mathrm{mM}$ glucose that increased to $8 \mathrm{mM}$ at the oligomycin step, $4 \mathrm{mM}$ glutamine, and $0.5 \mathrm{mM}$ sodium pyruvate. a. RGC density in 3-, 6-, and 10-month-old D2 and D2G mouse retina decreased significantly with age in the D2 retina (ANOVA, $F_{2,42}=15.96$, $* * * * p<0.0001$ ). The 10 -month-old D2 retinas had significantly lower RGC density than age-matched D2G retinas ( $t$ test, $\left.{ }^{* * *} p=0.0006\right)$. b Baseline oxygen consumption rate (OCR), normalized to protein per well, in the 10 -month-old D2G and D2 ON. There was no statistical difference across strain. c OCR in 10-month-old D2 and D2G ON with fluorocitrate (FC) treatment indicates significantly higher baseline OCR in the D2 $\left(t\right.$ test, $\left.{ }^{*} p=0.0445\right)$. Data are normalized

to the OCR with FC treatment and expressed as percent of FC OCR. d OCR in 10-month-old D2 and D2G ON in the presence of FC and oligomycin shows significantly lower OCR in the D2 ON compared to the D2G $(t$ test, $* p=0.034)$. e ATP production (the difference between oligomycin and FC baseline OCR) is significantly higher in the D2 ON at 10 months of age ( $t$ test, ${ }^{*} p=0.027$ ). f OCR with FCCP treatment in the presence of $\mathrm{FC}$ is significantly lower in the $\mathrm{D} 2$ compared to D2G ON at 6 months of age ( $t$ test, $* * p=0.0057)$. $g$ OCR after treatment with FCCP, in the presence of FC, shows significantly lower OCR in 10-month D2 ON compared to D2G ( $t$ test, $* p=0.028$ ). h The fraction of basal respiration driving ATP synthesis, or coupling efficiency, is significantly higher in the 10 -month D2 ON $(t$ test, $* * p=0.003)$ 
a

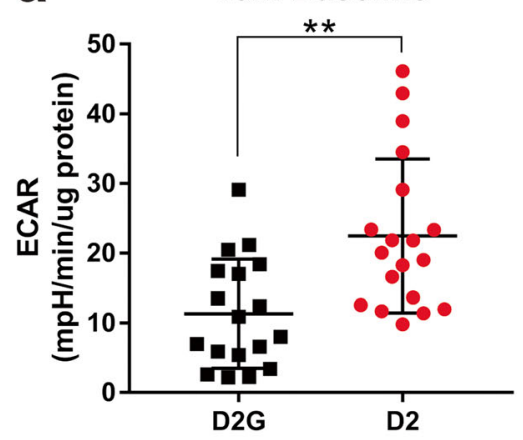

b

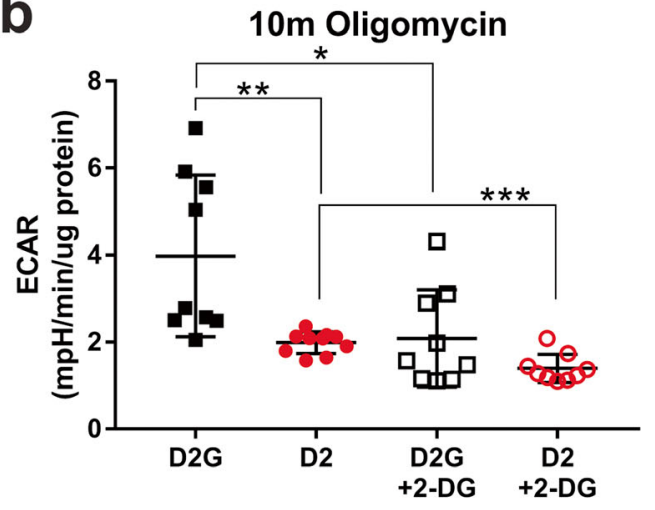

C

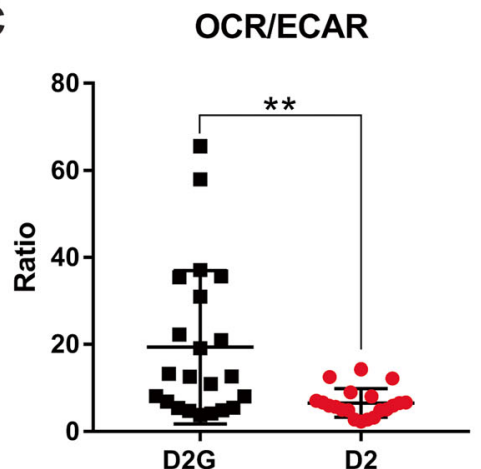

Fig. 6 ECAR in D2 and D2G ON in the presence of FC. a Baseline ECAR is significantly higher in the 10 -month-old D2 ON compared to the $\mathrm{D} 2 \mathrm{G}$, prior to FC ( $t$ test, $* * p=0.0012$ ). b ECAR with oligomycin treatment is significantly lower in the 10-month-old D2 ON compared to the D2G $\left(t\right.$ test, $\left.{ }^{* *} p=0.0011\right)$; ECAR from D2 ON with 2-deoxyglucose (2-DG) is significantly lower than D2 ON in glucose $(t$ test, $* * * p=$ 0.0003 ); and ECAR for D2G ON with 2-DG is significantly lower than $\mathrm{D} 2 \mathrm{G}$ ON in glucose $(t$ test, $* * p=0.0079)$. $\mathbf{c}$ The ratio of OCR to ECAR in the 10-month-old ONs shows the D2 has significantly lower ratio than the $\mathrm{D} 2 \mathrm{G}(t$ test, $* * p=0.0042)$

to quickly to changing energy conditions, especially if ATP production by oxidative phosphorylation is blocked.

The ratio of OCR to ECAR is used to show overall preference for oxidative phosphorylation or glycolysis. In Fig. 6c, the OCR to ECAR ratio is significantly higher in the D2G ON, demonstrating that the $\mathrm{D} 2 \mathrm{G} \mathrm{ON}$ has a much higher preference for oxidative phosphorylation than the D2 ON.

\section{Discussion}

This study set out to determine neurodegeneration-associated changes in mitochondrial respiration and glycolysis in the $\mathrm{ON}$. In the experiments without fluorocitrate, we observed decreased ATP production with progression of optic neuropathy in the D2 glaucoma model. These alterations may be a result of mitochondrial defect, or alternatively, the decreases in glucose transporter GLUT1 and monocarboxylate transporter MCT2 that occur in the 10-month-old D2 ON [3]. Interestingly, the largest decrease in ATP production in the D2 ON occurred at the 6-month time point, the same age at which MCT2, the monocarboxylate transporter specific to the RGC axon, is significantly decreased in the D2 ON. MCT2 functions to bring monocarboxylates like lactate, pyruvate, and ketone bodies into the axon. Lack of substrate availability is one contributor to decreased ATP production [29]. Another source of decreased ATP production is likely the diminished activity of electron transport chain complexes I, III, and IV that were observed as early at 5 months of age in the D2 ON [31]. Also, at 6 months of age in the D2 ON, AMPK activation is significantly increased, indicating the $\mathrm{ON}$ requires more energy than it is able to obtain [3]. These findings were corroborated by the lower ATP production observed in the D2 ON here.

OCR after oligomycin, FCCP, and antimycin A treatments did not vary across strain by age in the experiments run without fluorocitrate. The modest drop in OCR with oligomycin treatment for both strains suggests that mitochondrial ATP production does not contribute a significant degree to overall ATP production. Alternatively, it may indicate that glycolysis is predominant in this context. The decreased ATP production within the D2 strain, then, suggests reliance on glycolysis. Interestingly, a recently available RNA-sequencing dataset generated by the isolation of RGCs from D2 and D2G retina [10] showed upregulation of mRNA for key glycolysis enzymes in 9-month-old D2 RGCs, including hexokinase-1, aldolase $\mathrm{A}$, and lactate dehydrogenase $(\mathrm{LDH}) \mathrm{A}$. We have also observed LDH-A mRNA upregulation in the 10-month D2 ON (data not shown). The mRNA data is suggestive of increased glycolysis in the D2 RGCs and their axons. Interestingly, the $10 \mathrm{~m} \mathrm{D} 2 \mathrm{ON}$ has significantly decreased lactate levels as compared to the age-matched D2G (7). Low lactate in an $\mathrm{ON}$ that appears to be dependent upon glycolysis suggests that the lactate byproduct from glycolysis may be used as fuel by cells requiring substrate.

The average OCR after FCCP treatment exceeded the baseline OCR until 10 months of age for both the D2 and the D2G $\mathrm{ON}$. This suggests an aging effect on maximal respiration. The lack of a significant increase in OCR with FCCP treatment suggests that aging and oxidative damage have impaired the electron transport chain, as corroborated by one other study [31]. Substrate supply can also impact maximal respiration, as 
can metabolic enzymatic rates. As mentioned above, substrate supply is likely compromised in D2 ON because of decreased GLUT1 and MCT2 [3].

Decreased spare respiratory capacity, or the ability of a cell to meet increased ATP demand, was observed in both strains with aging, but the decrease was statistically different across age for the D2 ON. Conditions of severe stress, including oxidative stress or ischemia, and decreased substrate availability can deplete the spare respiratory capacity [32]. Oxidative stress has been documented in the D2 ON [33], and ischemia may be implicated in normal tension glaucoma [34]. Higher basal OCR with decreased spare capacity is a combination previously observed in trigeminal nucleus brain sections from a model of migraine [11].

\section{Fluorocitrate Findings}

The FC experiments endeavored to eliminate the contribution of astrocyte and oligodendrocyte mitochondria through preferential uptake of the FC by these cells and inhibition of aconitase [24], thereby providing a more sophisticated view of mitochondrial function in the axons. In the presence of FC, baseline OCR is higher in the 10-month D2 ON. The greater drop in OCR with oligomycin treatment in the D2 ON indicated more ATP production in the D2 as well. Given the increased fission [8] and greater signs of damaged/dysfunctional mitochondria in the D2 ON at this age [7, 9], it is surprising to find high OCR and greater ATP production in the D2 ON. It is possible, though, that the axonal mitochondria exhibit high OCR and ATP output because they must compensate for the dysfunctional among them. It may also be the case that pathological processes have selected for the survival of particularly efficient mitochondria. Noteworthy is that the D2 ON at 10 months is undergoing tremendous change, including reductions in axon action potential [6], mild to moderate loss of axons [5], and glial hypertrophy [35, 36]. Glial growth would increase energy demand, though these needs would not be met by axonal mitochondrial respiration. We have shown in a past study that ATP content of 10-month-old D2 $\mathrm{ON}$ is significantly decreased compared to 6-month D2 ON, and the higher the IOP of the animal, the lower the ATP content [6]. The Seahorse-based observations of high ATP production in 10-month-old D2 ON are nevertheless consistent with decreased overall ATP content as previously shown because (1) we are comparing 10-month-old D2 ON OCR to 10month-old D2G without the contribution of glial respiration, and (2) we showed that OCR in the presence of oligomycin declines significantly with age in the D2. OCR decline in the presence of oligomycin is generally attributable to ATP production, and is modified by proton leak and nonmitochondrial respiration.

High oxygen consumption, as observed here for the 10month D2 ON, can also come from non-mitochondrial respiration or proton leak. However, we determined that proton leak was not different between the glaucoma and control mice at 10 months. The 10 -month D2 ON mitochondria were more highly coupled than the $\mathrm{D} 2 \mathrm{G}$, also suggesting that proton leak was not contributing to the higher OCR.

Lower maximal respiration was observed in the D2 ON, starting at 6 months and extending to $10 \mathrm{~m}$. Since maximal respiration is primarily determined by substrate supply and oxidation, lower maximal respiration corroborates the potentially compromised substrate delivery expected in the D2 ON that has lower levels of GLUT1 and MCT2 [3], restricting substrate getting across the plasma or mitochondrial membrane [29]. The number of mitochondria and/or the cristae density can also limit maximal respiration. As shown by our research [7, 9] and others [37], mitochondria have compromised cristae and smaller size in the D2 ON. Despite the lower maximal respiration, the 10-month D2 ON nevertheless had a significantly higher ratio of ATP-linked respiration to maximal respiration. The higher ratio of ATP-linked respiration indicates that functioning mitochondria in the D2 ON axons were more efficiently producing ATP; however, since the ratio was less than 1 for both D2 and D2G (with FC) at 10 months but the D2 was significantly higher, the D2 ON is working closer to its maximum output capacity than the D2G. The high oxygen consumption for ATP production in the D2 may exist to compensate for the glaucoma-related changes in the $\mathrm{ON}$ that contribute to energy depletion, including compromised access to lactate and increased levels of activated AMPK [3].

The cut ends of axons, created by chopping the ON into pieces for the assay, could be exposed to fluorocitrate, potentially allowing the compound to interfere with axonal oxidative phosphorylation. OCR fell by $\sim 50 \mathrm{pmol} / \mathrm{min} / \mu \mathrm{g}$ protein with the addition of fluorocitrate, a level that represented between 25 and $30 \%$ of the baseline OCR, suggesting that its impact on OCR is consistent with expectations for blocking astrocyte, but little of the axonal, OCR.

\section{ECAR Analysis}

Extracellular acidification rate (ECAR) at baseline was significantly higher in the 6- and 10-month D2 compared to the D2G ON (without FC). Glycolysis upregulation, evident in both strains by the positive difference in ECAR with oligomycin treatment over baseline, may compensate for failing mitochondria in aged D2 and D2G ON. The high ECAR values in the D2 ON suggest higher levels of glycolysis in D2 than D2G ON. We had determined that GLUT1 levels were significantly decreased in 10-month-old D2 ON; higher glycolysis may account for the significantly lower levels of glycogen also observed in 10month-old D2 ON [3] if those pools are being utilized at the expense of incoming substrate. However, as discussed below, glycolysis appears to comprise a smaller fraction than expected of the ECAR output in D2 ON. 
In the presence of FC, ECAR at baseline was significantly higher in the 10-month $\mathrm{D} 2 \mathrm{ON}$ than the D2G. An initial interpretation of such an observation would be significantly higher glycolysis in the 10-month D2 ON. However, ECAR represents proton release from glycolysis as well as TCA cycle substrate oxidation. The ratio of one to the other varies by cell type and context [30]. It was only in the fluorocitrate experiments that we were able to estimate the contribution of respiration to ECAR. Roughly $30 \%$ of the D2 ON ECAR fell when 2-DG was the substrate in the media instead of glucose, suggesting that $70 \%$ of the ECAR under these conditions was derived from the $\mathrm{HCO}_{3}{ }^{-}+\mathrm{H}^{+}$given off by TCA cycle $\mathrm{CO}_{2}$. These figures likely overestimate the contribution of substrate oxidation because glycolysis was not completely inhibited with 2-DG in this context due to the residual glucose in the media. In addition, endogenous sources of glucose, such as glycogen, can serve as a source for glucose in the presence of 2-DG and thus, contribute to ECAR. This may explain why, in contrast to the D2 ON, ECAR in the D2G ON was evenly split (50-50) between glycolysis and respiration. D2G ON maintains significantly higher glycogen stores than D2 ON, for which stores are depleted with glaucoma pathogenesis [3]. These estimates of the source of ECAR assists our interpretation of the ECAR data. For the FC experiments, glial respiration was constrained. The lack of change in ECAR between baseline and FC treatment indicated that glial respiration did not contribute in an appreciable way to ECAR levels. On one level, this meets expectations of glia as glycolytic cells [38]. However, astrocyte mitochondria comprise the majority of mitochondrial mass in the $\mathrm{ON}$ [39], providing astrocytes with significant capacity for oxidative phosphorylation. In one analysis of glucose versus lactate substrate utilization in brain cortex, astrocytes oxidized $50 \%$ of the interstitial lactate and $35 \%$ of the glucose, while neurons oxidized no more than $50 \%$ of the lactate and $65 \%$ of the glucose found in the parenchyma [27]. These findings imply that cortical astrocytes preferentially utilize oxidative phosphorylation. Despite significant mitochondrial mass in the ON, these data suggest glial cell mitochondria nevertheless do not appear to contribute significantly to ECAR through substrate oxidation.

Axonal mitochondria respiration comprises a major portion of the ECAR, but this is likely context dependent. It was only in the fluorocitrate experiments that we were able to estimate ECAR contribution from glycolysis versus substrate oxidation. The D2 ON axonal mitochondria at 10 months of age were exceptionally efficient and productive compared to the D2G ON mitochondria. Oxidizing substrate would have increased ECAR, and did so to a larger degree in D2 than D2G. It is not surprising, then, that $\sim 70 \%$ of $\mathrm{D} 2 \mathrm{ON}$ ECAR could be attributable to axonal mitochondria respiration. D2 ON residual functional mitochondria have to compensate for degenerative changes and an apparent glycolysis limit. An additional piece of corroborating evidence is the significant decline in lactate levels in the 10-m D2 ON compared to D2G (7); low lactate either indicates that glycolysis is decreased, or that the lactate is being utilized as fuel in the D2 ON more so than in the $\mathrm{D} 2 \mathrm{G}$.

An important finding for the metabolic contribution to D2 optic neuropathy is that oligomycin in the presence of FC did not increase ECAR to the degree that it did in the D2G ON. The ECAR boost in the D2G ON with oligomycin treatment was double the ECAR observed in the D2 ON at 10 months of age. This indicates that the D2 ON was not capable of boosting glycolysis to meet ATP demand as well as the D2G $\mathrm{ON}$. When challenged with ATPase inhibition, D2G switches to glycolysis; but the 10-month D2 ON was not as capable at glycolysis upregulation. Since we have indications that ECAR, for the $\mathrm{D} 2 \mathrm{ON}$, is primarily from axonal mitochondria respiration, these data corroborate the limited ability of the D2 $\mathrm{ON}$ at 10 months to increase respiration with FCCP treatment. This lack of a true boost to glycolysis in the D2 ON with oligomycin could be a result of glucose uptake challenges inherent in an ON in which the GLUT1 protein levels were significantly decreased [3], or an indication of some constraint on glycolysis, such as inhibition of a rate-limiting enzyme or saturation of glycolytic flux. Phosphofructokinase, a ratelimiting enzyme in glycolysis, can be inhibited by citrate, a TCA cycle intermediate, as well as ATP. If oxidative phosphorylation is proceeding as well as indicated in the D2 ON with FC, then it is possible that glycolysis would be inhibited. However, that inhibition should have been relieved by oligomycin treatment. As previously discussed, mRNA for a number of important glycolytic enzymes are significantly upregulated in the D2 RGCs compared to either younger D2 or RGCs taken from D2G mice [10]. Further analysis of enzyme protein levels and activities will enable greater insight into the balance of metabolism in the degenerating D2 visual system.

\section{RGC and Visual Function Indices Do Not Predict Metabolic Changes}

As shown in the scatterplots of RGC density or visual acuity by respiration, mice with fewer RGCs or lower visual acuity did not demonstrate significantly greater deficits in OCR than other mice within their group. This result is attributable to many things, including the mixed population of cells and axons in the $\mathrm{ON}$ that can obscure bioenergetic analysis, the presence of degenerating axons in the D2 10-month-old samples, and the high variability of OCR data. It is important to remember that OCR is a readout of mitochondrial function, not mitochondrial number or dynamics. However, ON populations did reveal significant differences of metabolic management between the glaucomatous and control ON, including an inability to metabolically switch to glycolysis when called upon, and an untenable reliance on oxidative phosphorylation. 
Acknowledgements The authors would like to thank Nate Pappenhagen for tissue processing and quantification analysis and Kayla Trautman and Amelia McMullen for overall technical assistance.

Authors' Contributions AHJ, LC, and MHR collected data, contributed to the writing, and reviewed the paper; PK designed the study, analyzed and interpreted data, and reviewed the paper; YRC interpreted data and reviewed the paper; DMI designed the study, analyzed data, created figures, and wrote the paper. All authors read and approved the final manuscript.

Funding Information This work is supported by the National Institutes of Health EY-026662 to DMI.

\section{Compliance with Ethical Standards}

Ethical Approval All applicable international, national, and/or institutional guidelines for the care and use of animals were followed.

Conflict of Interest The authors declare that they have no conflict of interest.

Open Access This article is distributed under the terms of the Creative Commons Attribution 4.0 International License (http:// creativecommons.org/licenses/by/4.0/), which permits unrestricted use, distribution, and reproduction in any medium, provided you give appropriate credit to the original author(s) and the source, provide a link to the Creative Commons license, and indicate if changes were made.

\section{References}

1. MacVicar BA, Newman EA (2015) Astrocyte regulation of blood flow in the brain. Cold Spring Harb Perspect Biol 7:1-15. https:// doi.org/10.1101/cshpespect.at020388

2. Inman DM, Harun-Or-Rashid M (2017) Metabolic vulnerability in the neurodegenerative disease glaucoma. Front Neurosci 11: https://doi.org/10.3389/fnins.2017.00146

3. Harun-or-Rashid M, Pappenhagen N, Wilson GN et al (2018) Structural and functional rescue of chronic metabolically stressed optic nerves through respiration. J Neurosci 38:5122-5139. https:// doi.org/10.1523/JNEUROSCI.3652-17.2018

4. Simpson IA, Carruthers A, Vannucci SJ (2007) Supply and demand in cerebral energy metabolism: the role of nutrient transpoters. J Cereb Blood Flow Metab 27:1766-1791. https://oi.org/10.1038/ sj.jcbfm. 9600521

5. Libby RT, Anderson MG, I-HH P et al (2005) Inherited glaucoma in DBA/2J mice: pertinent disease features for studying the neurodegeneration. Vis Neurosci 22:637-648. https://doi.org/10.1017/ S0952523805225130

6. Baltan S, Inman DM, Danilov CA, Morrison RS, Calkins DJ, Horner PJ (2010) Metabolic vulnerability disposes retinal ganglion cell axons to dysfunction in a model of glaucomatous degeneration. J Neurosci 30:5644-5652. https://doi.org/10.1523/JNEUROSCI. 5956-09.2010

7. Kleesattel D, Crish SD, Inman DM (2015) Decreased energy capacity and increased autophagic activity in optic nerve axons with defective anterograde transport. Investig Ophthalmol Vis Sci 56: 8215-8227. https://doi.org/10.1167/iovs.15-17885

8. Ju W-K, Kim K-Y, Lindsey JD, Angert M, Duong-Polk KX, Scott RT, Kim JJ, Kukhmazov I et al (2008) Intraocular pressure elevation induces mitochondrial fission and triggers OPA1 release in glaucomatous optic nerve. Invest Ophthalmol Vis Sci 49:49034911. https://doi.org/10.1167/iovs.07-1661

9. Coughlin L, Morrison RSS, Horner PJ, Inman DM (2015) Mitochondrial morphology differences and mitophagy deficit in murine glaucomatous optic nerve. Invest Ophthalmol Vis Sci 56: 1437-1446. https://doi.org/10.1167/iovs.14-16126

10. Williams PA, Harder JM, Foxworth NE et al (2017) Vitamin B3 modulates mitochondrial vulnerability and prevents glaucoma in aged mice. Science 760:756-760. https://doi.org/10.1120/science. aal0092

11. Fried NT, Moffat C, Seifert EL, Oshinsky ML (2014) Functional mitochondrial analysis in acute brain sections from adult rats reveals mitochondrial dysfunction in a rat model of migraine. AJP Cell Physiol 307:C1017-C1030. https://doi.org/10.1152/ajpcell. 00332.2013

12. Gorgun FM, Zhuo M, Singh S, Englander EW (2014) Neuroglobin mitigates mitochondrial impairments induced by acute inhalation of combustion smoke in the mouse brain. Inhal Toxicol 26:361-369. https://doi.org/10.1080/10810730902873927.Testing

13. Kooragayala K, Gotoh N, Cogliati T, Nellissery J, Kaden TR, French S, Balaban R, Li W et al (2015) Quantification of oxygen consumption in retina ex vivo demonstrates limited reserve capacity of photoreceptor mitochondria. Investig Ophthalmol Vis Sci 56: 8428-8436. https://doi.org/10.1167/iovs.15-17901

14. Hassel B, Paulsen RE, Johnsen a FF (1992) Selective inhibition of glial cell metabolism in vivo by fluorocitrate. Brain Res 576:120 124

15. John SW, Smith RS, Savinova OV, Hawes NL, Chang B, Turnbull D, Davisson M, Roderick TH et al (1998) Essential iris atrophy, pigment dispersion, and glaucoma in $\mathrm{DBA} / 2 \mathrm{~J}$ mice. Invest Ophthalmol Vis Sci 39:951-962

16. Anderson MG, Smith RS, Hawes NL, Zabaleta A, Chang B, Wiggs JL, John SWM (2002) Mutations in genes encoding melanosomal proteins cause pigmentary glaucoma in $\mathrm{DBA} / 2 \mathrm{~J}$ mice. Nat Genet 30:81-85

17. Inman DM, Sappington RM, Horner PJ, Calkins DJ (2006) Quantitative correlation of optic nerve pathology with ocular pressure and corneal thickness in the DBA/2 mouse model of glaucoma. Invest Ophthalmol Vis Sci 47:986-996. https://doi.org/10. 1167/iovs.05-0925

18. Jakobs TC, Libby RT, Ben Y, John SWM, Masland RH (2005) Retinal ganglion cell degeneration is topological but not cell type specific in DBA/2J mice. J Cell Biol 171:313-325

19. Prusky GT, West PW, Douglas RM (2000) Behavioral assessment of visual acuity in mice and rats. Vis Res 40:2201-2209

20. Prusky GT, Silver BD, Tschetter WW, Alam NM, Douglas RM (2008) Experience-dependent plasticity from eye opening enables lasting, visual cortex-dependent enhancement of motion vision. J Neurosci 28:9817-9827. https://doi.org/10.1523/JNEUROSCI. 1940-08.2008

21. Wong AA, Brown RE (2012) A neurobehavioral analysis of the prevention of visual impairment in the DBA/2J mouse model of glaucoma. Invest Ophthalmol Vis Sci 53:5956-5966. https://doi. org/10.1167/iovs.12-10020

22. Wong AA, Brown RE (2007) Age-related changes in visual acuity, learning and memory in $\mathrm{C} 57 \mathrm{BL} / 6 \mathrm{~J}$ and DBA/2J mice. Neurobiol Aging 28:1577-1593. https://doi.org/10.1016/j.neurobiolaging. 2006.07.023

23. Crish SD, Sappington RM, Inman DM, Horner PJ, Calkins DJ (2010) Distal axonopathy with structural persistence in glaucomatous neurodegeneration. Proc Natl Acad Sci U S A 107: 5196-5201. https://doi.org/10.1073/pnas.0913141107

24. Fonnum F, Johnsen A, Hassel B (1997) Use of fluorocitrate and fluoroacetate in the study of brain metabolism. Glia 21:106-113 
25. Paulsen RE, Contestabile A, Villani L, Fonnum F (1987) An in vivo model for studying function of brain tissue temporatily devoid of glial cell metabolism: the use of fluorocitrate. J Neurochem 48: $1377-1385$

26. Voloboueva LA, Suh SW, Swanson RA, Giffard RG (2007) Inhibition of mitochondrial function in astrocytes: implications for neuroprotection. J Neurochem 102:1383-1394. https://doi.org/ 10.1111/j.1471-4159.2007.04634.x

27. Zielke HR, Zielke CL, Baab PJ, Tyson Tildon J (2007) Effect of fluorocitrate on cerebral oxidation of lactate and glucose in freely moving rats. J Neurochem 101:9-16. https://doi.org/10.1111/j. 1471-4159.2006.04335.x

28. Dengler-Crish CM, Smith MA, Inman DM et al (2014) Anterograde transport blockade precedes deficits in retrograde transport in the visual projection of the DBA / 2J mouse model of glaucoma. Front Neurosci 8:290

29. Divakaruni AS, Paradyse A, Ferrick DA et al (2014) Analysis and interpretation of microplate-based oxygen consumption and $\mathrm{pH}$ data. Methods Enzymol 547:275-307

30. Mookerjee SA, Goncalves RLS, Gerencser AA, Nicholls DG, Brand MD (2015) The contributions of respiration and glycolysis to extracellular acid production. Biochim Biophys Acta Bioenerg 1847:171-181. https://doi.org/10.1016/j.bbabio.2014.10.005

31. Cwerman-Thibault H, Lechauve C, Augustin S, Roussel D, Reboussin É, Mohammad A, Degardin-Chicaud J, Simonutti M et al (2017) Neuroglobin can prevent or reverse glaucomatous progression in DBA/2J mice. Mol Ther - Methods Clin Dev 5:200220. https://doi.org/10.1016/j.omtm.2017.04.008

32. Hill BG, Dranka BP, Zou L, Chatham JC, Darley-Usmar VM (2009) Importance of the bioenergetic reserve capacity in response to cardiomyocyte stress induced by 4-hydroxynonenal. Biochem $\mathrm{J}$ 424:99-107. https://doi.org/10.1042/BJ20090934

33. Inman DM, Lambert WS, Calkins DJ, Horner PJ (2013) $\alpha$-Lipoic acid antioxidant treatment limits glaucoma-related retinal ganglion cell death and dysfunction. PLoS One 8:e65389. https://doi.org/10. 1371/journal.pone.0065389

34. Caprioli J, Coleman AL (2010) Blood pressure, perfusion pressure, and glaucoma. Am J Ophthalmol 149:704-712. https://doi.org/10. 1016/j.ajo.2010.01.018

35. Bosco A, Breen KT, Anderson SR, Steele MR, Calkins DJ, Vetter ML (2016) Glial coverage in the optic nerve expands in proportion to optic axon loss in chronic mouse glaucoma. Exp Eye Res 150: 34-43. https://doi.org/10.1016/j.exer.2016.01.014

36. Cooper ML, Crish SD, Inman DM, Horner PJ, Calkins DJ (2016) Early astrocyte redistribution in the optic nerve precedes axonopathy in the DBA/2J mouse model of glaucoma. Exp Eye Res 150:22-33. https://doi.org/10.1016/j.exer.2015.11.016

37. Ju W, Kim K, Noh YH et al (2015) Increased mitochondrial fission and volume density by blocking glutamate excitotoxicity protect glaucomatous optic nerve head astrocytes. Glia 63:736-753. https://doi.org/10.1002/glia.22781

38. Supplie LM, Düking T, Campbell G, Diaz F, Moraes CT, Götz M, Hamprecht B, Boretius S et al (2017) Respiration-deficient astrocytes survive as glycolytic cells in vivo. J Neurosci 37:4231-4242. https://doi.org/10.1523/JNEUROSCI.0756-16.2017

39. Perge JA, Koch K, Miller R, Sterling P, Balasubramanian V (2009) How the optic nerve allocates space, energy capacity, and information. J Neurosci 29:7917-7928. https://doi.org/10.1523/ JNEUROSCI.5200-08.2009

40. Mosconi L, Pupi A, De Leon MJ (2008) Brain glucose hypometabolism and oxidative stress in preclinical Alzheimer's disease. Ann N Y Acad Sci 1147:180-195. https://doi.org/10. 1196/annals.1427.007.Brain

41. Vlassenko AG, Vaishnavi SN, Couture L, Sacco D, Shannon BJ, Mach RH, Morris JC, Raichle ME et al (2010) Spatial correlation between brain aerobic glycolysis and amyloid- $\beta(A \beta)$ deposition. Proc Natl Acad Sci 107:17763-17767. https://doi.org/10.1073/ pnas. 1010461107

42. Pellerin L, Magistretti PJ (1996) Excitatory amino acids stimulate aerobic glycolysis in astrocytes via an activation of the $\mathrm{Na}+\mathrm{K}+$ ATPase. Dev Neurosci 18:336-342. https://doi.org/10.1159/ 000111426

43. Lindsay KJ, Du J, Sloat SR et al (2014) Pyruvate kinase and aspartate-glutamate carrier distributions reveal key metabolic links between neurons and glia in retina. Proc Natl Acad Sci 111:1557915584. https://doi.org/10.1073/pnas. 1412441111

Publisher's Note Springer Nature remains neutral with regard to jurisdictional claims in published maps and institutional affiliations. 